\title{
ATOMIC HYDROGEN SURVEY NEAR THE GALACTIC PLANE
}

\author{
B. F. BURKE, E. T. ECKLUND, J. W. FIROR, \\ H. E. TATEL, ${ }^{*}$ AND M. A. TUVE \\ Department of Terrestrial Magnetism, Carnegie Institution, \\ Washington, D.C., U.S.A.
}

Using an 8-meter Würzburg parabola on an equatorial mount in Washington, D. C., we have completed about half of the necessary observations and reductions for a survey of the $1420-\mathrm{Mc} / \mathrm{s}$ hydrogen emission for all sky areas near the galactic plane which are visible here.

Our observed areas are centered on 010,013 , and 017 degrees and on similar points for every 10 degrees of longitude from $l=317$ through 0 degrees to $l=220$ degrees; and they have been centered on a galactic plane slightly tilted with respect to the plane $b=0$. Thus in the region to each side of $l=360$ degrees we have centered our cross section on $b=-1$ degree; from $l=040$ degrees to $l=110$ degrees we have centered on $b=0$, and for $l=113$ to beyond 220 degrees we have centered on $b=+1$ degree. Near the plane we observe at 2-degree intervals, increasing to 5 -degree intervals beyond $b= \pm 10$ degrees. The elliptical antenna beam has not been set parallel to the Galaxy, but it has been left continuously with the long axis of the ellipse in the E-W position. The antenna beamwidth, to indicated half-power points on each side, is 21 by $3 \frac{1}{2}$ degrees as measured on the sun. Since some saturation of the detector occurred on the sun, the true beam area is of slightly smaller dimensions, probably 2 by 3 degrees. Sky settings are made within $\pm 2 / 10$ degrees in right ascension and $\pm 1 / 10$ degree in declination. The sky-pointing dials were checked on the sun to within $\pm 1 / 10$ degree. Frequency calibrations are within $\pm 4 \mathrm{kc} / \mathrm{s}$, giving hydrogen velocities to $\pm 1 \mathrm{~km} / \mathrm{second}$.

In this paper we show the curves obtained for the galactic plane, and give one or two samples of cross sections across the plane.

\section{DESCRIPTION OF THE APPARATUS}

Our 54-channel $\mathrm{H}$-line receiver is diagramed in Fig. 1. Each channel is 12 $\mathrm{kc} / \mathrm{s}$ wide to half-power points. Channel 27 is $2.000 \mathrm{Mc} / \mathrm{s}$ and the channels are spaced at intervals of $18.953 \mathrm{kc} / \mathrm{s}$ both up and down to cover a $1-\mathrm{Mc} / \mathrm{s}$ band. We thus have $4-\mathrm{km} / \mathrm{second}$ intervals in hydrogen Doppler velocity between centers of adjacent channels. The local oscillators (near $1393 \mathrm{Mc} / \mathrm{s}$ ), which were used from 1957 January through 1958 June, were self-excited single-tube free oscillators, keyed by triodes in their plate circuits and monitored against

* Deceased 1957 November 15. 


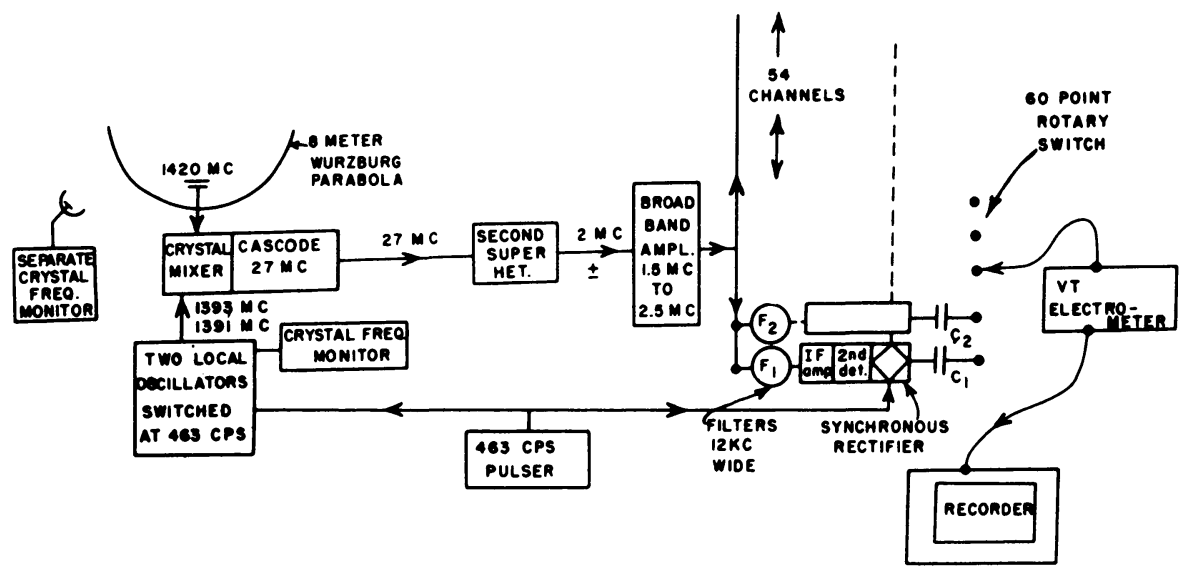

FIG. 1. Block diagram of 54-channel $\mathrm{H}$-line receiver.

a crystal harmonic reference frequency. The $\mathrm{H}$-frequency oscillator was on automatic servo-control against this crystal harmonic; the comparison frequency oscillator (1.5 Mc/s distant) was manually set each hour or oftener. An independent crystal oscillator radiating a harmonic $(1420.3603 \mathrm{Mc} / \mathrm{s})$ from the laboratory window toward the parabolic dish was used hourly or oftener as an over-all check. Frequency settings are known to about one-fourth channel or $\pm 1 \mathrm{~km} / \mathrm{second}$ for this series of observations. (At this writing, early 1958 July, the self-oscillators are being replaced by crystal-controlled oscillators with the usual doublers and triplers.) Our antenna feed needs revision, because the crystal mixer is 6 wavelengths from the dipole (behind the dish). Standing-wave ratios are 1.10 to 1.20 for both sidebands.

The noise figure of the receiver and the corresponding temperature calibration are measured at intervals with a Bendix noise diode, accepting the manufacturer's correction $(0.70)$ for transit time at $1400 \mathrm{Mc} / \mathrm{s}$. The temperature calibration differs from our old figures obtained on our old sweep-scan receiver of several years ago. We have not sought out the cause of this difference. We find $T=102 \pm 2^{\circ} \mathrm{K}$ for the maximum at $l=147, b=0$, and $T=99 \pm 3^{\circ} \mathrm{K}$ for $l=50, b=0$. These sky points are used for our routine gain checks. The over-all gain is reasonably constant, at times remaining within about 5 percent for 2 to 4 weeks, but showing abrupt and larger changes as vacuum tubes fail. The mixer crystals degrade slightly in use, but abrupt and large degradation of noise figure occurs only a few times per year. The crystals we now use (Philco 1N263) give a very satisfactory noise figure of 3.2 to 4.5. We have no automatic gain control, so added noise enters only as an increase in statistical fluctuation, and noise figure changes of a few per cent are ignored. Gain changes or diode drifts in a few troublesome single channels among the 54 are encountered. The hot diodes in the audio-frequency synchronous rectifier circuits give some trouble with drifts of $2 / 3$ to 2 millivolts $\left(2\right.$ to $6^{\circ} \mathrm{K}$ ) and require rebalancing every few 
days to keep the measurements within $\pm 1{ }^{\circ} \mathrm{K}$ without applying individual corrections. The over-all gain is such that $100^{\circ} \mathrm{K}$ sky temperature on one channel observed for 4.8 minutes gives 33 millivolts on the output condenser of that channel, which is "sampled" by the electrometer. Our standard procedure is to return each output condenser to zero after each sampling by the rotary electrometer switch, thus making each 4.8-minute run independent of past runs.

The precision of single observations of 4.8 minutes' duration on each single channel of the 54 -channel set was found to be $\pm 2.4^{\circ} \mathrm{K}$, with the averages of three observations showing a statistical r.m.s. fluctuation of $\pm 1.5^{\circ} \mathrm{K}$ (probable error $\pm 1.0^{\circ} \mathrm{K}$ ). This same result was obtained for runs taken two months apart on cold areas of the sky. This is in reasonable agreement with the input circuit equivalent noise temperature of around $1000^{\circ} \mathrm{K}$ indicated by the Bendix diode.

Perhaps the most disconcerting and difficult variable has been the position of the "zero line," namely, the position of the recorder pen for zero extra radiation on the $1420 \mathrm{Mc} / \mathrm{s}$ band when compared with the 1418-Mc/s (sometimes $1422-\mathrm{Mc} / \mathrm{s}$ ) channel comparison-frequency band in the sky. The recorder should draw a statistically straight line $\left( \pm 2.4^{\circ} \mathrm{K}\right)$, having no slope with frequency, since it records the 54 channels covering a $1-\mathrm{Mc} / \mathrm{s}$ band around $1420.4 \mathrm{Mc} / \mathrm{s}$. This zero line, however, is very sensitive to slight changes in the impedance of the dipole-and-flat-reflector that illuminates the dish at the focus of the parabola. We have found almost hourly monitoring necessary in order to know the position of indicated zero hydrogen for the low, medium, and high groups of channels. The "zero line" for channels 0 to $54(1 \mathrm{Mc} / \mathrm{s}$ total spread) is a straight line, but it may slope up or down, and it may shift position by $1^{\circ} \mathrm{K}$ or as much as $3^{\circ} \mathrm{K}$ during a few hours (especially when the weather or the temperature changes rapidly). The slope is usually less than $2{ }^{\circ} \mathrm{K}$ in going from channel 1 to channel 54 , but at times (snow or rain) it is as great as $5^{\circ} \mathrm{K}$ from end to end. Some of our records are shown in the figures, and a long "slash" at the sides of each record shows each end of the zero line for $0^{\circ} \mathrm{K}$ (no hydrogen; cold sky area) for that hour of observation.

Although our parabolic dish is located only 4 miles north from downtown Washington, we have experienced detectable interference (from sources outside of our own set, which has several crystal harmonic "markers") on only four occasions. Twice we have had I. F. pickup on single channels (from strong nearby broadcasting stations) and twice we have unwittingly pointed the antenna very near the sun (behind the clouds). No other sources of interference down to $\pm 2.4^{\circ} \mathrm{K}$ per single observation have been detectable.

\section{DESCRIPTION OF RECORDS}

A typical original record is shown in Fig. 2 (original record from Brown recorder). The "sky zero" line $\left(0^{\circ} \mathrm{K}\right.$ of hydrogen $)$ is indicated by the long marks or slashes near the beginning and end of the record. This zero line is determined daily or oftener by looking at cold areas of the sky, and by 


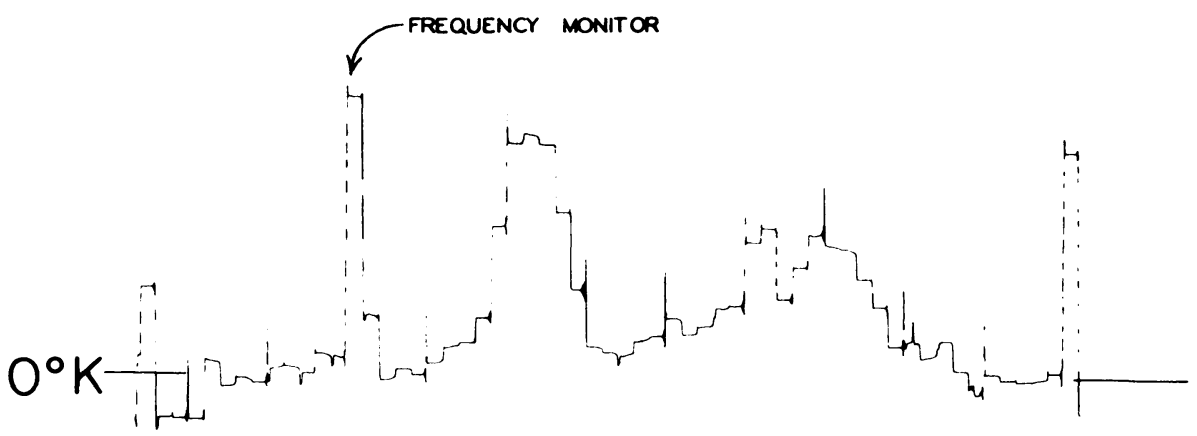

FIG. 2. Original record from Brown recorder at $l=217^{\circ}, b=-1^{\circ}$ with signal from frequency monitor visible.

ignoring the middle channels which show some hydrogen. An example is given in Fig. 3. Calibration marks (100 millivolts direct on the electrometer, and 100 millivolts on one condenser for the the duration of the observation) are at beginning and end of the record. The position of $\mathrm{H}_{0}=1420.406 \mathrm{Mc} / \mathrm{s}$ is marked after reference to the frequency monitor.

For each sky position (with dish on sidereal drive) three or four records are made at a selected center frequency, and then the frequency is shifted $3 \frac{1}{2}$ channels (at local oscillator of the second superheterodyne) for a second group of points, which interlaces the first, thus giving $2-\mathrm{km} / \mathrm{second}$ velocity spacings between observed frequencies for the two groups of points. Some features of the sky have velocity spreads so narrow that this procedure has been valuable, since our channels are only $2.2 \mathrm{~km} / \mathrm{second}$ wide (to half-power). The whole group of 6 or 8 records corresponding to this one sky position is then transferred to one sheet of translucent graph paper, giving a single record of the kind shown in Fig. 3. Pertinent information is also transferred

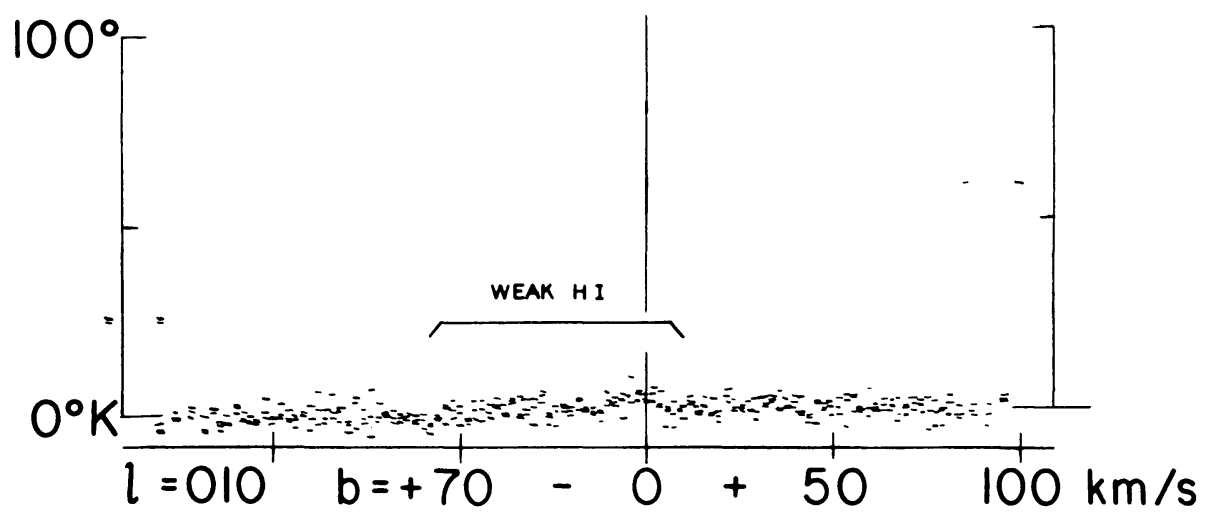

Fig. 3. Tracing of six records at $l=010^{\circ}, b=-70^{\circ}$ showing trace of hydrogen and cold sky background. 

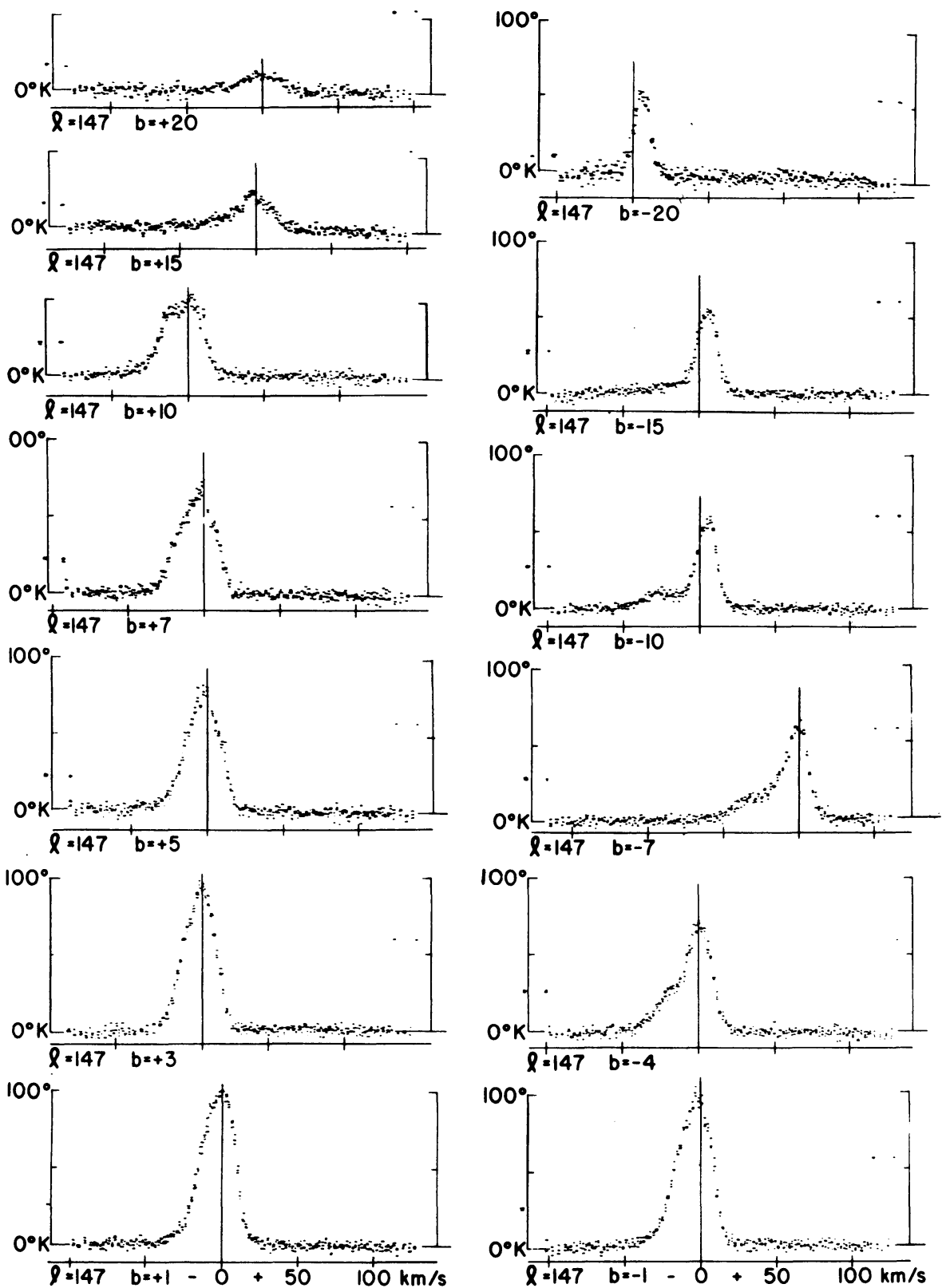

Fig. 4. A section at $l=147^{\circ}$.

to this sheet to give $H_{R}$, the channel frequency on the graph for hydrogen at rest (corrected to local standard of rest) for the particular date, sky direction, and frequency settings in use for that particular set of records. Our sky-temperature sensitivity is usually left at a standard value $\left(20^{\circ} \mathrm{K}\right.$ per 

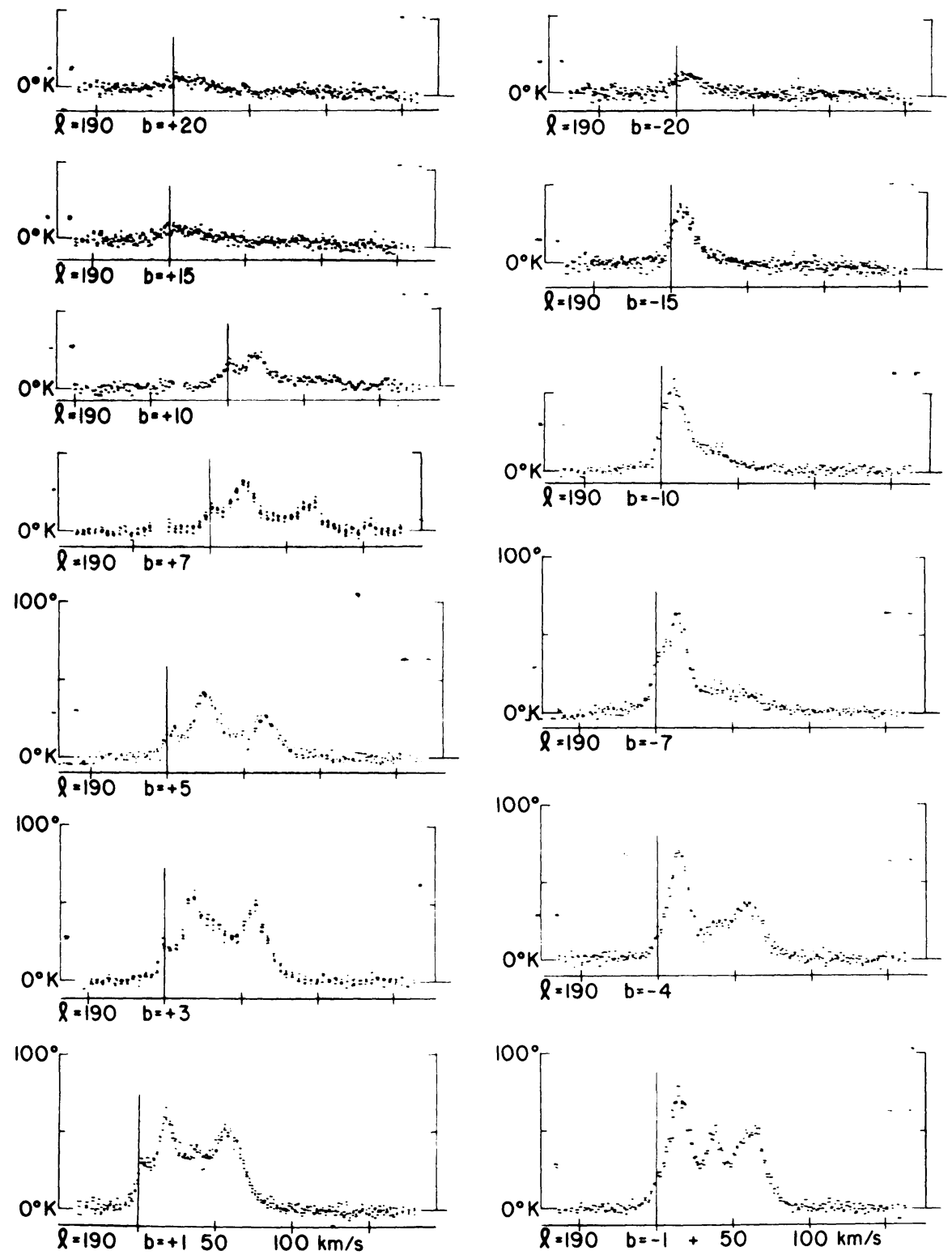

FIG. 5. A section at $l=190^{\circ}$.

inch of recorder deflection). The figure on the opposite page shows an assembly of such records, which represents a section taken across the plane of the galaxy at $l=147$ degrees. The figure above shows a similar cross section at $l=190$ degrees. 

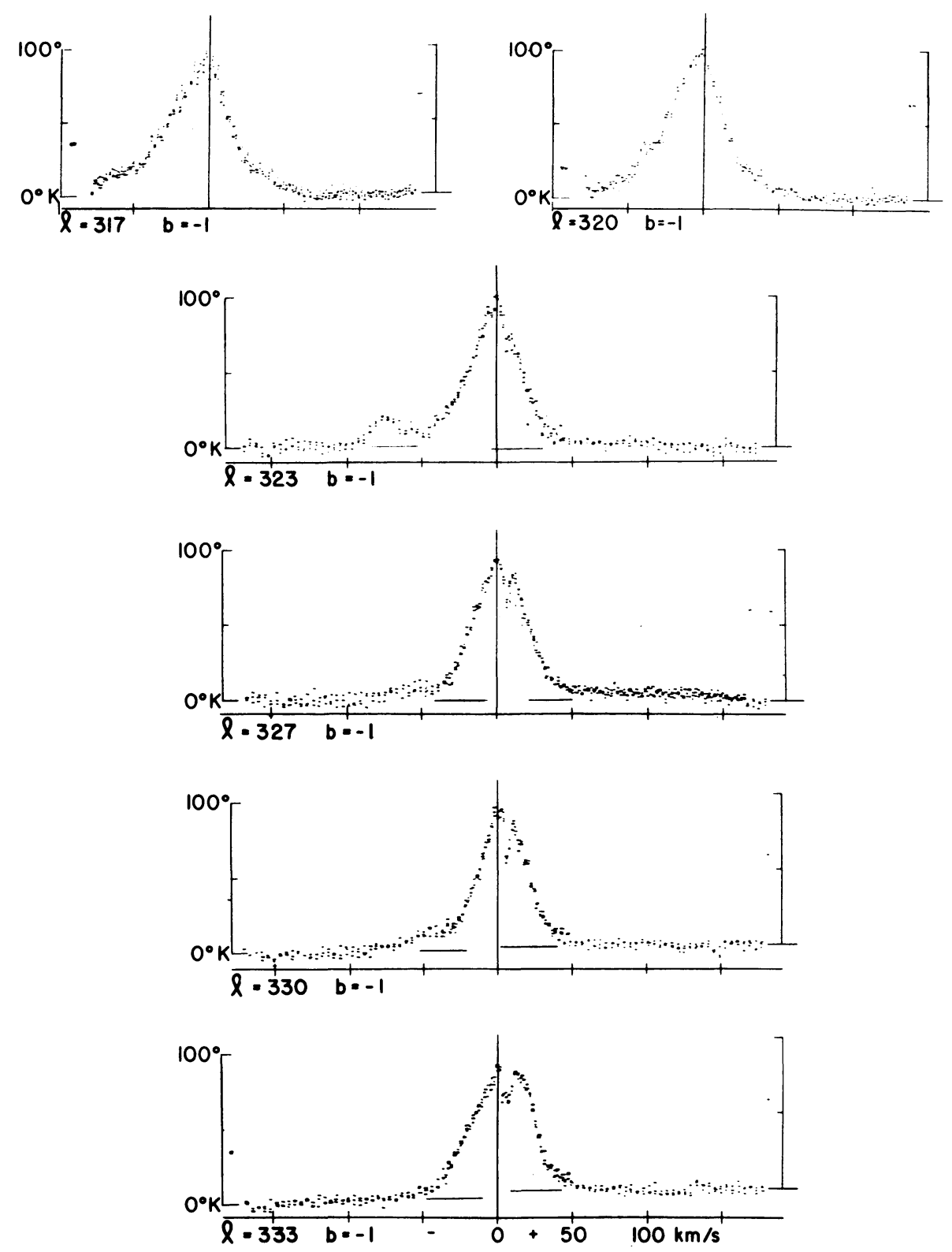

Frg. 6. Longitudes 317 to $330^{\circ}$.

The remaining succession of figures shows the records we have obtained for the vicinity of the galactic plane (we use a tilted plane as above indicated) for the regions visible from our site in Washington, D.C., namely, from $l=317$ through 0 degrees to $l=227$ degrees. 
NO. 72
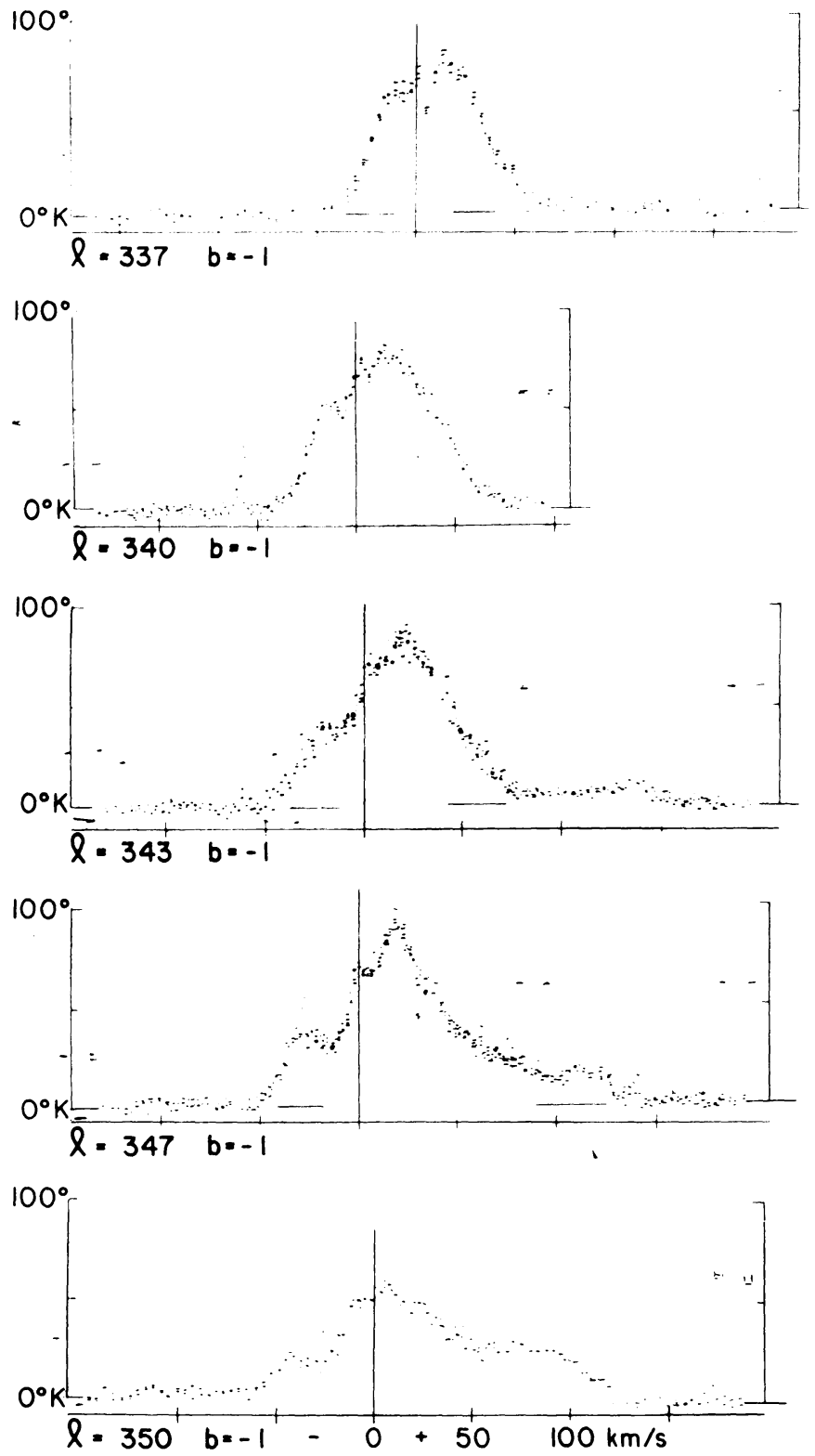

Fig. 7. Longitudes 337 to $350^{\circ}$.

We have to date made observations for about half of the positions selected as indicated above for our survey with this parabola from -20 to +20 degrees. A separate report from this laboratory is being made on the regions from +20 and -20 degrees to the galactic poles. 

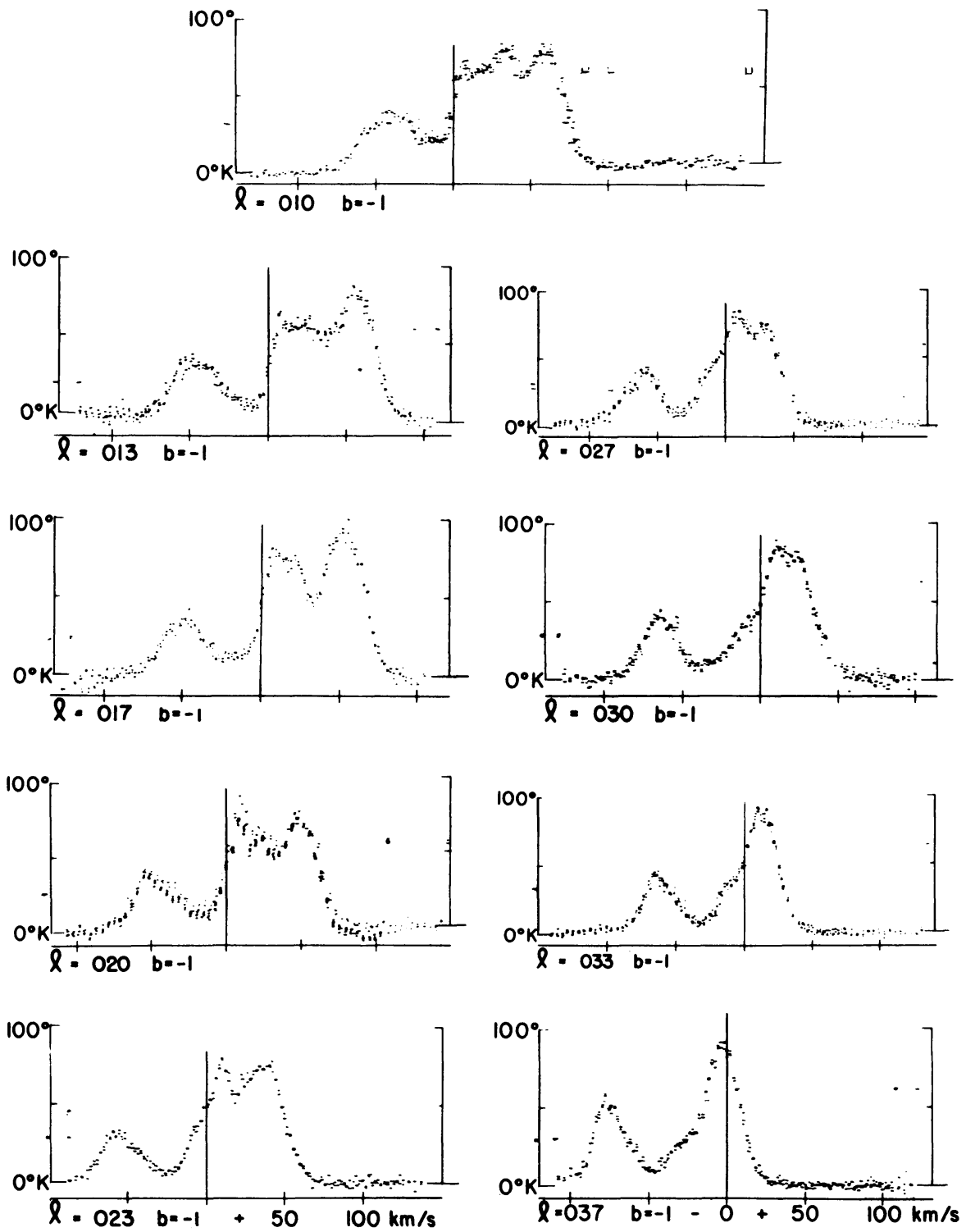

Fig. 8. Longitudes 353 to $007^{\circ}$. 
NO. 72

BURKE et al.

383
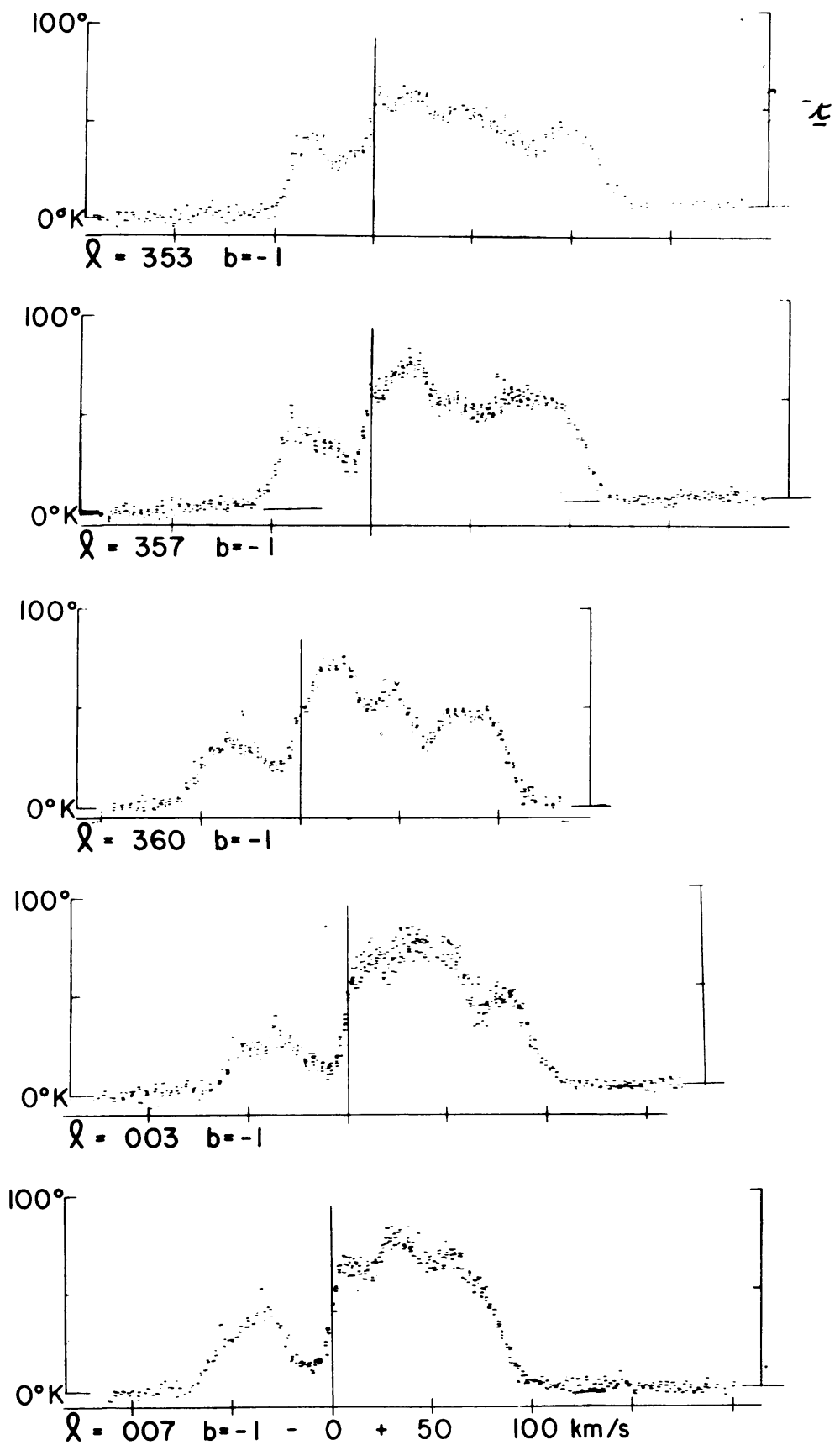

FIG. 9. Longitudes 010 to $037^{\circ}$. 

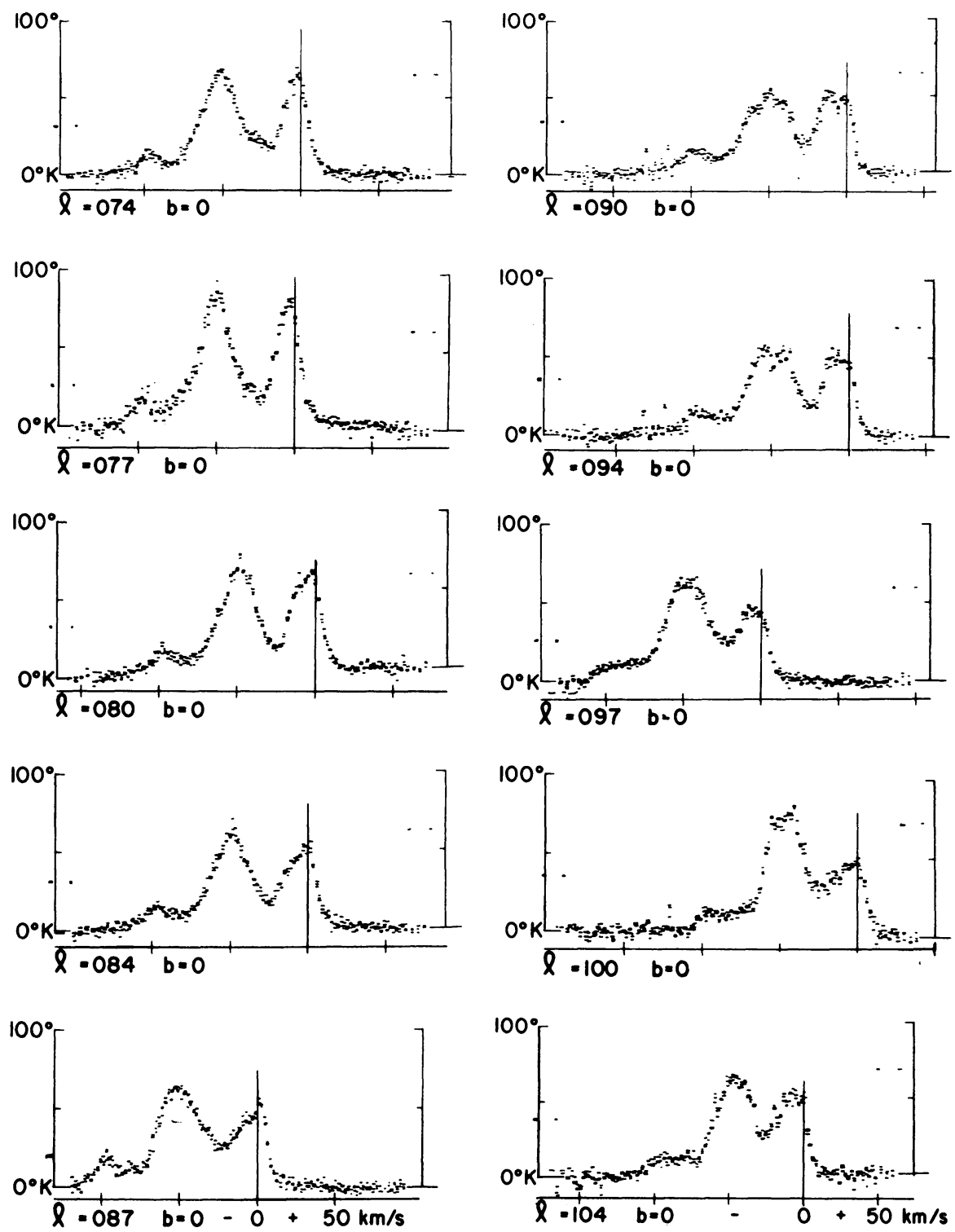

FIg. 10. Longitudes 040 to $070^{\circ}$. 

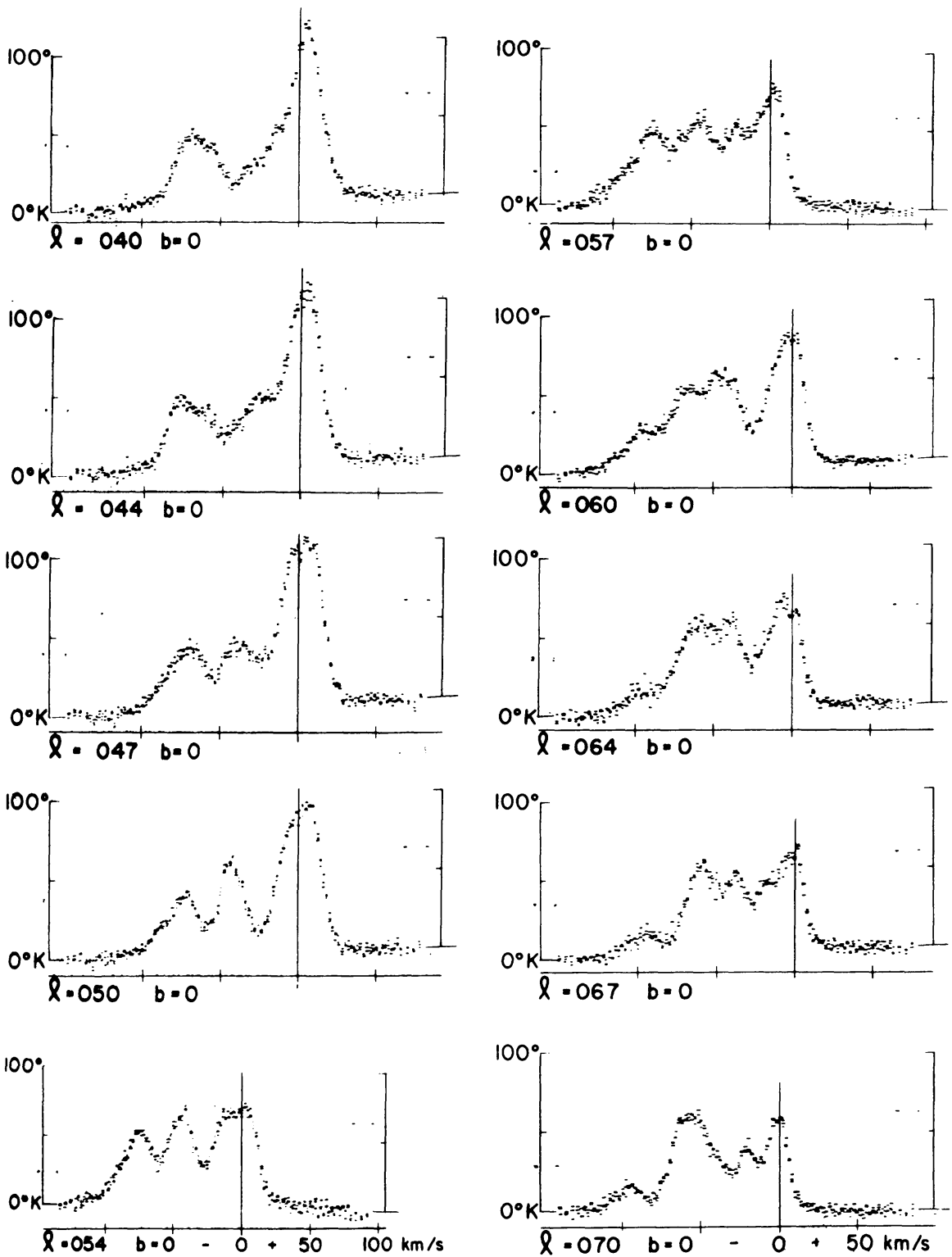

FIg. 11. Longitudes 074 to $104^{\circ}$. 

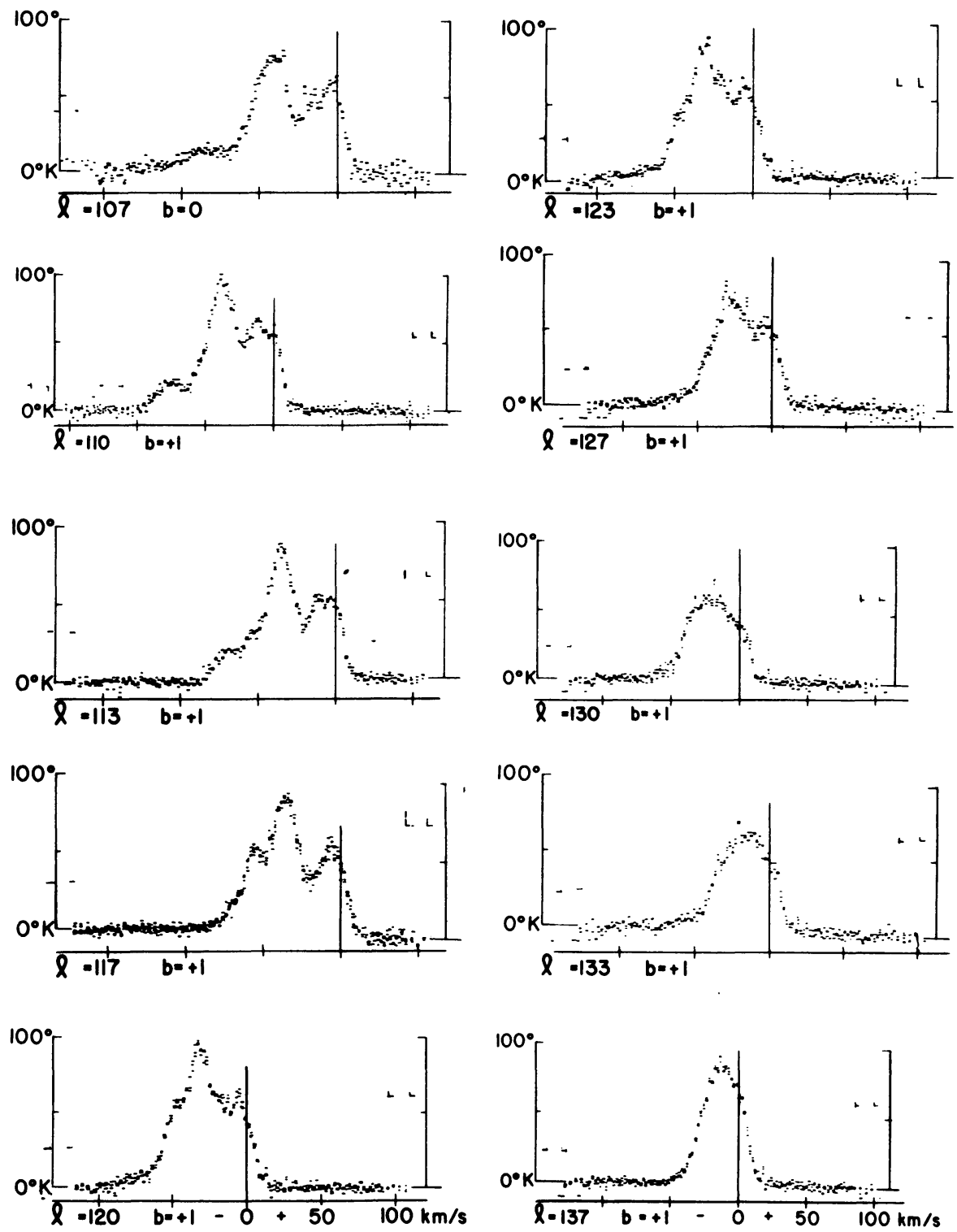

Fig. 12. Longitudes 107 to $137^{\circ}$. 
NO. 72
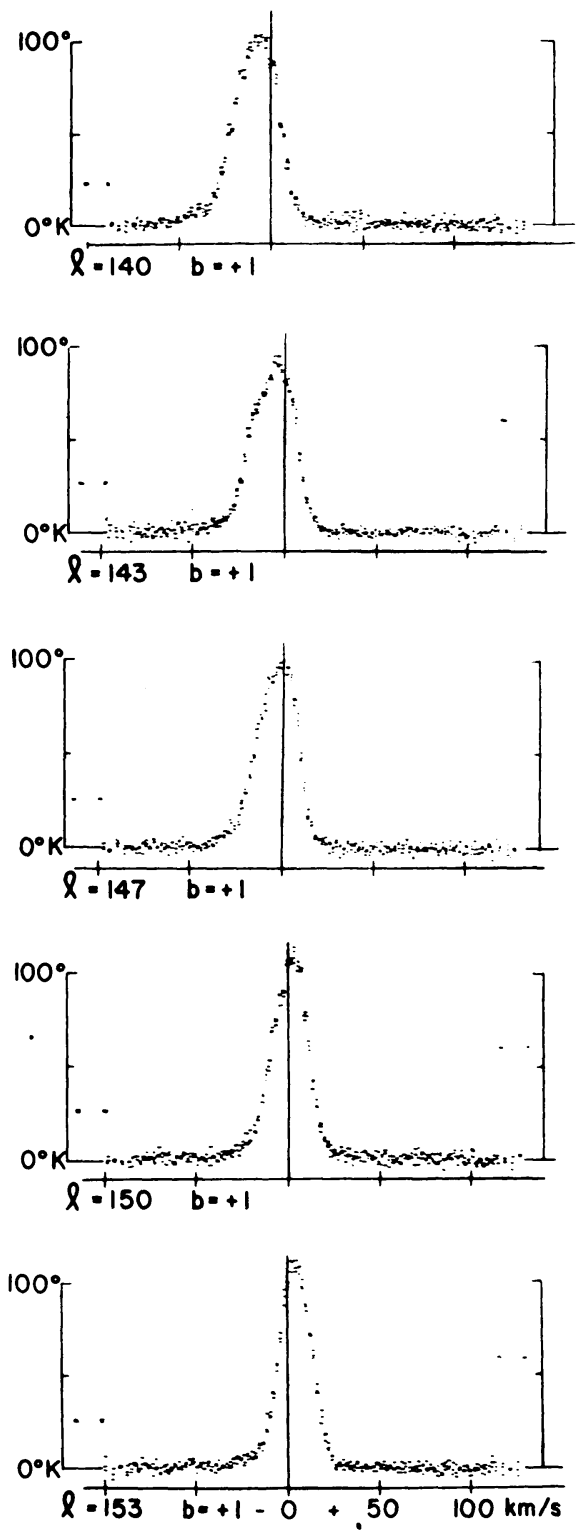
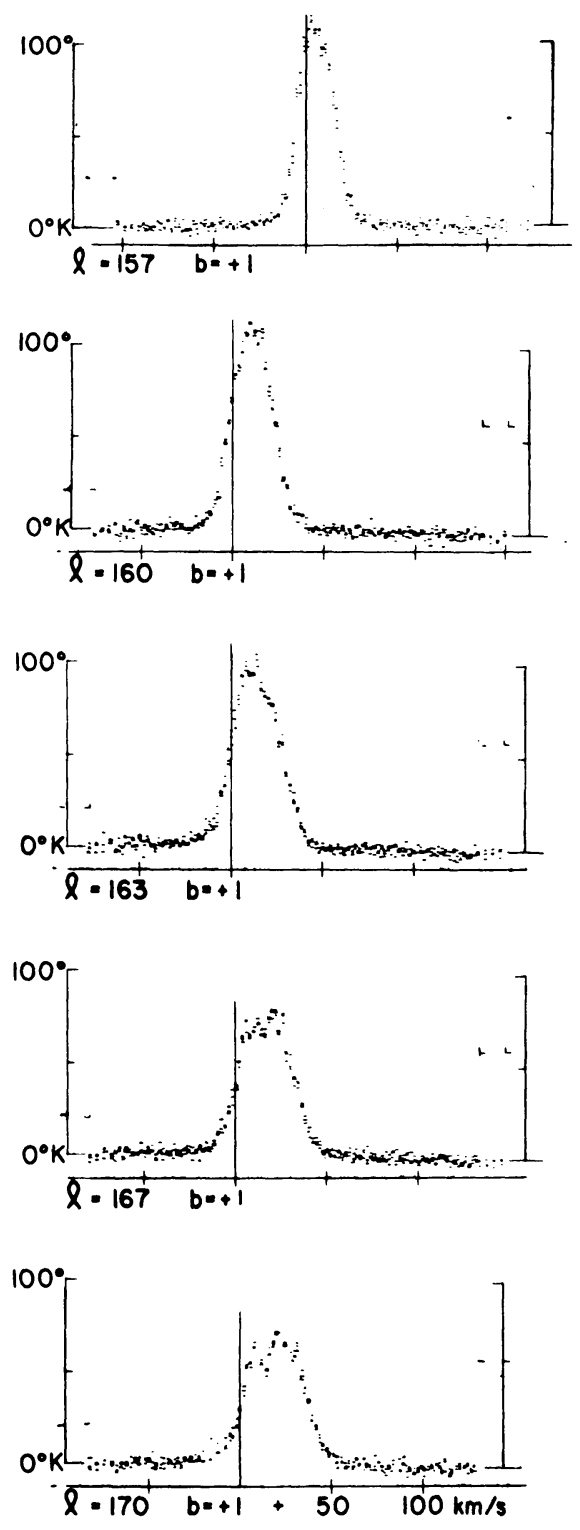

FIG. 13. Longitudes 140 to $170^{\circ}$. 
388

DISCRETE SOURCES

PT. III
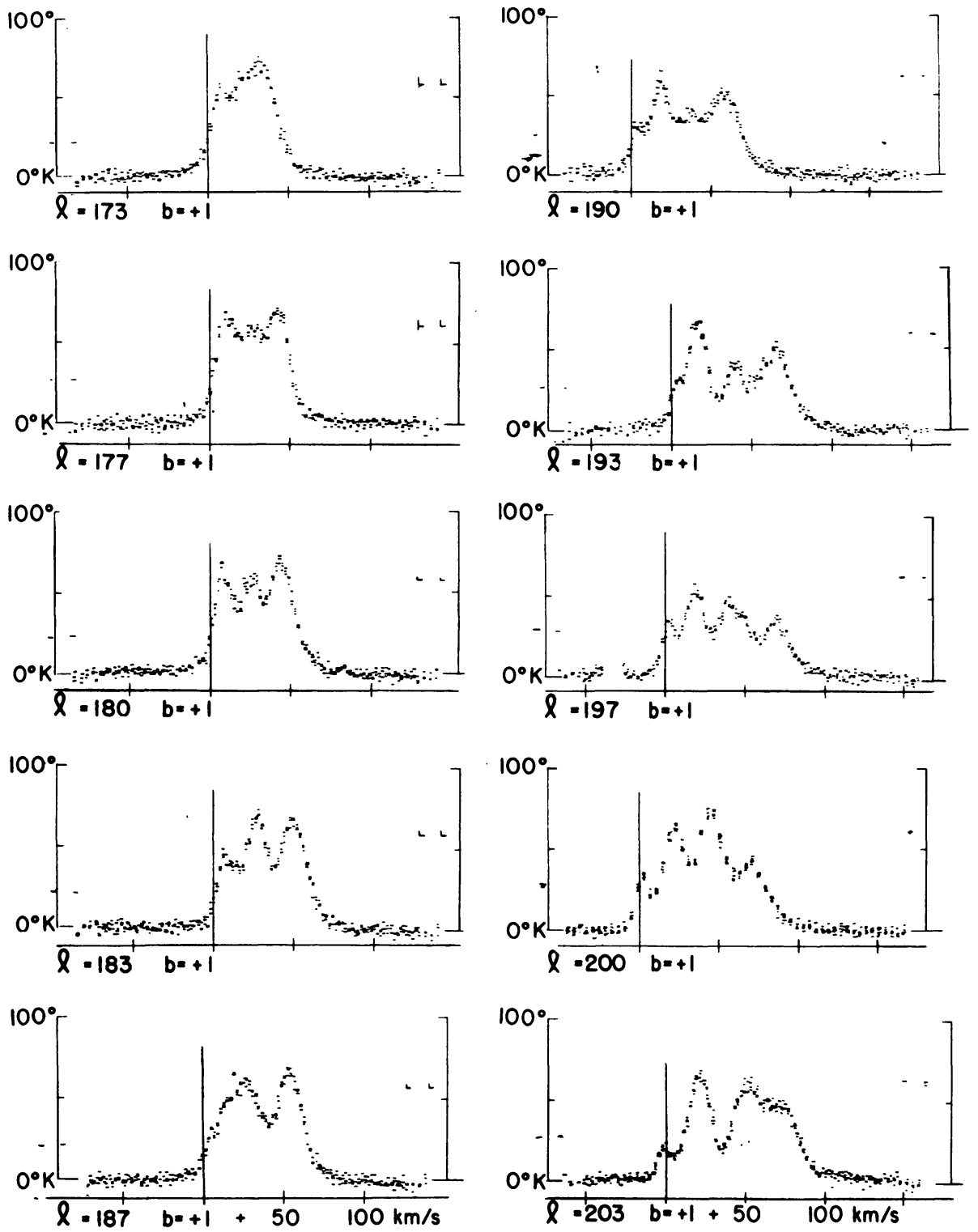

Fig. 14. Longitudes 173 to $203^{\circ}$.

https://doi.org/10.1017/S0074180900051184 Published online by Cambridge University Press 

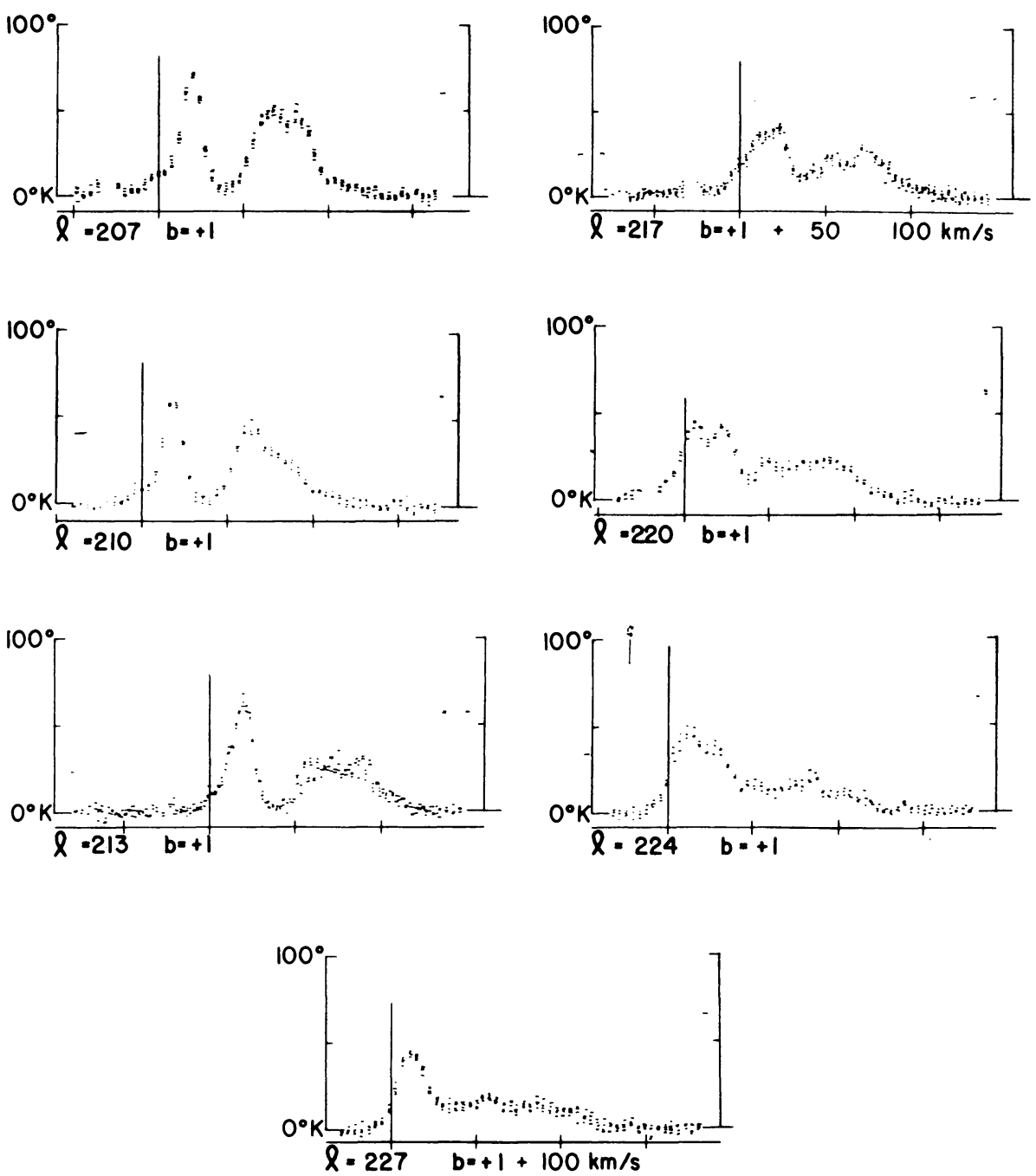

Fig. 15. Longitudes 207 to $227^{\circ}$. 